Accademia Gioenia di Catania

Anno di fondazione 1824
Boll. Accad. Gioenia Nat. Sci. (Catania)

53 (383), MISC4-MISC5 (2020)

doi:10.35352/v53i383.91

\title{
Allelopathic effects of Cynara cardunculus L. extracts*
}

\author{
Aurelio Scavo ${ }^{1 \dagger}$ \\ ${ }^{1}$ Department of Agriculture, Food and Environment, University of Catania, Italy
}

\begin{abstract}
Summary
Weeds are recognized as the most important biotic factor affecting yields in agroecosystems. The negative effects derived from the irrational use of herbicides have led to an increasing interest to eco-friendly practices for weed control, including the manipulation of allelopathic mechanisms. The present study aims to explore the potential use of Cynara cardunculus L. leaf extracts for the biological control of weeds and pathogen microorganisms. In a first trial, the allelopathic effects of its leaf aqueous extracts were demonstrated on seed germination of six common weeds. Secondly, the set-up of the most efficient extraction method of its allelochemicals in terms of costs, yields and inhibitory activity was realized, selecting dried leaves as the best plant material and ethanol and ethyl acetate as the best solvents. Moreover, new C. cardunculus allelochemicals (cynaratriol, desacylcynaropicrin, 11,13-dihydro-desacylcynaropicrin and pinoresinol) were purified. Third, the effect of genotype, harvest time and light stress (by plant shading) was evaluated on the phytotoxicity, quantity and composition of sesquiterpene lactones in C. cardunculus leaf extracts through a new UHPLC-MS/MS analysis method. Wild and cultivated cardoon showed the highest concentrations, while spring was the best harvest time. Moreover, light stress stimulated the synthesis of these allelochemicals. In a second trial, the effects resulting from 3-years cultivation, in two different areas, with globe artichoke, cultivated and wild cardoon were studied on the quali/quantitative composition of the weed soil seed bank and on soil eubacterial communities. In both areas, C. cardunculus reduced the weed seed bank size compared to controls. Nevertheless, the presence of cultivated cardoon had a negative influence towards Bacillus subtilis and a positive one on Pseudomonas putida and Azospirillum brasilense. Lastly, cultivated cardoon leaf extracts were assessed in vitro for the control of several microorganisms of agriculture and food interest. All the extracts showed an
\end{abstract}

*Abstract of one of the Best PhD Theses Award, 2020.

†E-mail: aurelio.scavo@unict.it 
important antimicrobial activity, especially when using ethanol as extracting solvent.

Keywords: Cynara cardunculus, herbicides, weed management, allelopathy.

\section{Riassunto}

\section{Effetti allelopatici degli estratti di Cynara cardunculus $L .{ }^{*}$}

Le piante infestanti sono riconosciute come il più importante fattore biotico che influenza i raccolti negli agroecosistemi. Gli effetti negativi derivati dall'uso irrazionale di erbicidi hanno portato ad un crescente interesse verso pratiche eco-compatibili per il controllo delle piante infestanti, compresa la manipolazione dei meccanismi allelopatici. Il presente studio si propone di esplorare il possibile uso di estratti di foglie di Cynara cardunculus L. per il controllo biologico di piante infestanti e di microorganismi patogeni. In un primo esperimento, sono stati provati gli effetti allelopatici di tali soluzioni acquose sulla germinazione dei semi di sei infestanti comuni. In secondo luogo, l'estrazione dei suoi allelochimici è stata realizzata secondo il metodo più efficiente in termini di costi, rese e attività inibitoria, individuando nelle foglie essiccate la parte della pianta con la resa migliore, e l'etanolo e l'acetato di etile come i migliori solventi. Inoltre, sono state ottenute e purificate nuove componenti allelochimiche del $C$. cardunculus (cinaratriolo, desacilcinaropicrina, 11,13-diidro-desacilcinaropicrina e pinoresinolo). In terzo luogo, è stato valutato l'effetto del genotipo, del tempo di raccolta e dello stress dovuto all'illuminazione (mediante ombreggiamento delle piante) sulla fitotossicità, quantità e composizione dei lattoni sesquiterpenici negli estratti di foglie di $C$. cardunculus attraverso un nuovo metodo di analisi UHPLC-MS / MS. Il cardo selvatico e il cardo coltivato hanno mostrato le concentrazioni più elevate, mentre la primavera è stata il periodo migliore per la raccolta. Inoltre, lo stress da illuminazione ha stimolato la sintesi di questi allelochimici. In un secondo esperimento sono stati studiati gli effetti derivanti dalla coltivazione di 3 anni, in due differenti aree, con carciofo, cardo coltivato e cardo selvatico sulla composizione qualitativa e quantitativa della banca semi del suolo delle infestaitni e sulle comunità eubatteriche del suolo. In entrambe le aree, il $C$. cardunculus ha ridotto le dimensioni della banca semi di infestanti rispetto ai campioni di controllo. Tuttavia, la presenza di cardo coltivato ha avuto un'influenza negativa nei confronti del Bacillus subtilis e positiva sulla Pseudomonas putida e sullo Azospirillum brasilense. Infine, estratti di foglie di cardo coltivati sono stati valutati in vitro per il controllo di diversi microrganismi di interesse agricolo e alimentare. Tutti gli estratti hanno mostrato un'importante attività antimicrobica, soprattutto quando si utilizza l'etanolo come solvente di estrazione.

Parole chiave: Cynara cardunculus, erbicidi, gestione delle piante infestanti, allelopatia.

*Riassunto di una delle Migliori Tesi di Dottorato premiate nel 2020. 